\title{
Water degrading effects on the bond behavior in FRP-strengthened masonry
}

\author{
Bahman Ghiassi $^{1}$, Giancarlo Marcari² ${ }^{2}$ Daniel V. Oliveira ${ }^{3}$, Paulo B. Lourenço ${ }^{4}$ \\ ISISE, University of Minho, Department of Civil Engineering, Guimarães, Portugal
}

\begin{abstract}
Fiber reinforced polymers are being extensively used for external strengthening of masonry structures. However, durability of this strengthening technique under environmental conditions is still under investigation. Previous studies indicate that moisture plays an important role in the durability of bond between FRP and substrate. Moisture can cause degradation in the bond behavior and also in the mechanical properties of the constituent materials. This paper presents and discusses the results of an experimental investigation on the effects of moisture on the bond behavior in FRP-strengthened masonry bricks. The degradation in the bond performance has been investigated by performing pull-off and pull-out tests on the conditioned specimens. The
\end{abstract}

${ }^{1} \mathrm{PhD}$ Student, ISISE, University of Minho, Department of Civil Engineering, Azurém, 4800-058 Guimarães, Portugal. Phone: +351 253510 499, fax: +351 253510 217, E-mail:

bahmanghiassi@civil.uminho.pt

${ }^{2}$ Assistant Researcher, University Sapienza of Rome, Department of Structural Engineering and Geotechnics, 00184 Rome, Italy. Phone: +39 06 44585399, fax: +39 06 44585399, E-mail: giancarlo.marcari@uniroma1.it

${ }^{3}$ Associate Professor, ISISE, University of Minho, Department of Civil Engineering, Azurém, 4800-058 Guimarães, Portugal. Phone: +351 253510 247, fax: +351 253510 217, E-mail: danvco@civil.uminho.pt

${ }^{4}$ Professor, ISISE, University of Minho, Department of Civil Engineering, Azurém, 4800-058 Guimarães, Portugal. Phone: +351 253510 209, fax: +351 253510 217, E-mail: pbl@civil.uminho.pt 
change in the mechanical properties of the materials has also been investigated. Comparative analysis has been performed and the results are presented and critically discussed.

Keywords: A. Polymer-matrix composites; B. Debonding; B. Environmental degradation; D. Mechanical testing; Masonry. 


\section{Introduction}

External strengthening of masonry structures with Fiber Reinforced Polymers (FRPs) has become popular in the last years. The efficacy and reliability of this strengthening technique depends intrinsically on the bond between the composite material and the masonry substrate. Although previous studies have shown the advantages of using FRP composites in strengthening structures, their long-term performance and durability are still unknown. Since the bond between FRP and substrate is a key mechanism in this strengthening technique, investigating its long-term behavior and durability is crucial for the performance prediction of the strengthened structure during its service life [1-2].

The most common environmental factors, which a structure is exposed to during its service life, are moisture and temperature variation, and alkaline and acidic environments. Since most of the environmental factors and deterioration processes are dependent on or coupled with moisture, a full understanding of moisture effects on deterioration of bond is a key step in durability modeling of FRP-strengthened masonry elements [3]. The effect of moisture on the durability of bond in FRP-strengthened concrete elements has been studied extensively, e.g. [3-6]. However, the available literature for FRP-strengthened masonry elements is still rare, e.g. [7-9]. Therefore, performing comprehensive experimental and numerical studies is mandatory for understanding the moisture induced degradation mechanisms in FRP-strengthened masonry.

The available results show that moisture exposure plays an important role in durability of bond in FRP applications, as it reduces the bond strength and fracture energy [3, 7, 10]. The failure mode often changes from cohesive to adhesive, and the force-displacement diagrams of the bond behavior show a non-linear trend at a lower applied force. These observations are usually attributed to extensive moisture plasticization of the polymer adhesive and additional breakage of 
interfacial bonds [6]. The moisture at the bond interface decreases the free surface energy and adhesion strength [11]. Moreover, it can produce vapor and osmotic pressure in the interface resulting in local debonding [11]. On the other hand, moisture absorption may affect the mechanical properties of epoxy resin, which has a direct effect on the adhesion and bond behavior. As discussed above, the information about the bond degradation is mostly obtained from investigations performed on FRP-strengthened concrete elements. However, the degrading effect of moisture on the bond behavior varies with material properties, surface treatments, and specimens configurations [7]. The significant mechanical and chemical difference between clay bricks and concrete may lead to different bond degradation mechanisms, which need further studies. The wide variety of FRP composites and epoxy resins used in structural applications complicates this issue. Therefore, performing an extensive experimental investigation on brick elements strengthened with FRP composites, which are conventionally used for strengthening masonry structures, is required for providing baseline experimental results and also for understanding the moisture degradation mechanisms in FRP-strengthened bricks.

This paper is devoted to investigating the effect of water on the bond behavior in FRPstrengthened masonry elements. Hand-made clay bricks, with low compressive strength, were used as representative of old bricks [12]. The clay bricks were strengthened with unidirectional glass fibers and immersed in water for 24 weeks after complete curing. The glass fibers were applied to the masonry brick surface with an epoxy resin following the wet lay-up procedure. The bond degradation due to moisture exposure is investigated by performing pull-off and pullout (single-lap shear bond) tests after different periods of water immersion. The changes in the bond behavior are investigated in terms of bond strength, bond stiffness, fracture energy and 
failure mode. It is noted that the change in mechanical properties of material constituents with water immersion is also investigated.

\section{Experimental program}

The experimental study addresses an investigation on the changes in the bond behavior of FRPstrengthened masonry bricks due to saturation (water immersion). The material characterization tests, specimens' preparation, moisture conditioning and post-ageing test methods are presented in this section.

\subsection{Materials characterization}

Mechanical tests have been performed on brick, epoxy resin, primer and GFRP coupon specimens to investigate the effect of moisture on the properties of material constituents, see Fig. 1Fig. 1. The tests are performed on specimens after different periods of water immersion. The mechanical properties of the unconditioned specimens are also evaluated to provide a baseline for the results obtained from the conditioned specimens. The tests have been performed according to the relevant test standards available and the results are presented as the mean value and coefficient of variation $(\mathrm{CoV})$ of five specimens, see Table 1Table 1 . It can be seen that the brick presents rather high CoVs (about 20\%), as it is hand-made and traditionally fired. The GFRP coupons exhibit also high CoVs (about 15\%) due the manufacture process, whereas the epoxy resin and primer exhibit lower CoVs (about 10\%).

\subsubsection{Masonry brick}

The mechanical properties of masonry bricks are obtained according to standards EN 772-1 (2002) [13] and UNI EN 8942-3 (1986) [14] in terms of compressive strength, $f_{\mathrm{cb}}$, tensile strength, $f_{\mathrm{tb}}$, and elastic modulus, $E_{\mathrm{b}}$. The compressive strength is obtained by performing 
compressive tests on $40 \mathrm{~mm}$ brick cubes, in the flatwise direction. The elastic modulus of bricks is determined by performing compressive tests on $120 \times 40 \times 40 \mathrm{~mm}$ brick specimens, in the lengthwise direction. It is expected that only moderate anisotropy is present [12]. Three point bending tests are performed on 160x40x40 mm brick specimens to obtain the horizontal flexural tensile strength.

\subsubsection{Epoxy resin and primer}

Mechanical properties of the epoxy resin and primer are determined by performing the tests on dog-bone shape specimens, see Fig. 1Fig. 1(c). Although seven days are proposed for curing of the epoxy resin in the technical datasheet provided by the manufacturer, the specimens were cured for 60 days at room temperature. Previous studies have shown that curing times much longer than the period proposed by the suppliers are necessary for complete curing of cold-cured epoxy resins [15-16]. Cross-linking reactions are still in progress before complete curing of the epoxy resin, meaning that the material is not in a stable condition and with its maximum mechanical properties. In order to accurately investigate the effect of water on the properties of epoxy resin, it is necessary to separate the water degrading effects and epoxy resin cross-linking reactions.

The specimens' preparation and tensile tests were conducted following ISO 527-1 (2012) [17]. The tests were carried out with an Instron testing machine at a displacement rate of $0.01 \mathrm{~mm} / \mathrm{min}$. Deformation of the specimens was monitored by a clip gauge placed on the middle of the specimens. The glass transition temperature $\left(T_{\mathrm{g}}\right)$ of the epoxy resin was obtained by means of the differential scanning calorimetry (DSC) method. The thermal scans were carried out between $5^{\circ} \mathrm{C}$ and $200^{\circ} \mathrm{C}$ with a heating rate of $10^{\circ} \mathrm{C} / \mathrm{min}$. The $T_{\mathrm{g}}$ was calculated as the mean value of 4 tests. 


\subsubsection{GFRP composite}

Regarding the composite materials, the specimens' preparation and tensile tests were conducted according to ISO 527-1 (2012) [17]. The mechanical properties are obtained in terms of tensile strength, $f_{\mathrm{tf}}$, and elastic modulus, $E_{\mathrm{f}}$. The GFRP coupons were prepared following the wet lay-up procedure according to the code specifications, Fig. 1Fig. 1(d). Throughout this study, the changes in tensile strength and elastic modulus of GFRP coupons are normalized to the specimens' thickness in accordance with ASTM D7565-10 [18]. As in wet lay-up procedures the specimens' thickness varies, normalizing the mechanical properties to thickness can provide an accurate baseline for comparison purposes [19].

\subsubsection{Water absorption}

Water absorption tests have been performed on constituent materials based on the gravimetric sorption method according to ASTM D 570-98 (2010) [20]. The specimens were immersed in deionized water at $23^{\circ} \mathrm{C}$ after initial drying. Five specimens were then taken from the water periodically, wiped and weighed to measure the absorbed water with time. The difference between the initial weight and the weight after conditioning represents the moisture mass uptake. This procedure is repeated until having water absorption lower than $1 \%$ of the dry weight in three sequential measurements. According to the Fick's second law, the moisture absorption behavior can be modeled with the following differential equation:

$$
\frac{\partial C}{\partial t}=-D \frac{\partial^{2} C}{\partial x^{2}}
$$

This equation provides the moisture concentration, $C$, at a distance $x$ from the contact surface as a function of time $t$ and moisture diffusion, $D$. An approximate solution of this equation is:

$$
\frac{M}{M_{\infty}}=\frac{4}{h} \sqrt{\frac{D t}{\pi}}
$$


where, $M$ is the moisture content at time $t, M_{\infty}$ is the equilibrium moisture content at saturation point, and $h$ is the specimen's thickness.

The moisture uptake behavior is presented in Fig. 2Fig. 2. The brick specimens have absorbed $10 \%$ mass of moisture upon saturation while epoxy resin and GFRP coupons absorbed $1.6 \%$ and $3.7 \%$, respectively. The brick absorbed water is corresponding to $17 \%$ open porosity, which is reasonably high, even if higher values are normal in hand-made bricks [12]. Open porosity is defined as the voids volume in which the water can penetrate due to immersion and can be calculated as follows:

$f=\frac{W_{s}-W_{d}}{V \rho_{w}}$

where, $f$ is the open porosity, $W_{\mathrm{s}}$ is the saturated weight, $W_{\mathrm{d}}$ is the dry weight, $V$ is the volume of the specimen, and $\rho_{\mathrm{w}}$ is the water density.

The moisture diffusion coefficient of epoxy resin and GFRP coupon have been determined by plotting the ratio $M / M_{\infty}$ against the square root of time, see Eq. (2). The calculated diffusion coefficients for epoxy resin and GFRP coupons immersed in deionized water are $8.85 \times 10^{-8}$ $\mathrm{mm}^{2} / \mathrm{sec}$ and $2.1 \times 10^{-7} \mathrm{~mm}^{2} / \mathrm{sec}$, respectively, in line with [21-22]. The equilibrium moisture content and moisture diffusion coefficient of the composite specimens are higher than of the epoxy resin. This difference is due to the imperfect interfaces that are in contact with water in the composite specimens [15].

Water absorption rate and the amount of absorbed water upon saturation in epoxy resins depend on different parameters such as formulation, curing conditions, environment temperature and humidity [15]. A wide range of equilibrium water content and moisture diffusion coefficients can be found in the literature. In $[5,22]$ a water content range from 0.42 to $1.88 \%$ was observed at 
saturation, with a moisture diffusion coefficient of $1.19 \times 10^{-9} \mathrm{~mm}^{2} / \mathrm{sec}$. In [15], a water content range from 4.7 to $8.7 \%$ was observed, with a moisture diffusion coefficient about $10^{-10} \mathrm{~mm}^{2} / \mathrm{sec}$.

\subsection{Specimens' preparation}

The GFRP sheets were applied to the brick surface following the wet lay-up procedure. The masonry bricks were dried in the oven before application of the GFRP sheets. After cleaning the brick surface, a two-part epoxy primer (MapeWrap Primer 1) was applied to the brick surface for preparation of the substrate surface before GFRP application. Finally, a two-part epoxy resin (MapeWrap 31) was used as matrix for the composite material and for adhesion to the masonry substrate.

For the pull-off tests, the specimens were prepared by application of GFRP sheets over a 180x80 $\mathrm{mm}^{2}$ area of the brick surface as shown in Fig. 3Fig. 3(a). For the pull-out tests, GFRP sheets of $50 \mathrm{~mm}$ width were applied on the brick surface. The bonded length of the strips was equal to 150 mm with a $40 \mathrm{~mm}$ unbonded part at the loaded end, see Fig. 3Fig. 3(b).

\subsection{Moisture conditioning}

The selected exposure is intended to investigate the influence of moisture on the bond behavior in FRP-strengthened masonry components. The test specimens were immersed in deionized water at a temperature of $23^{\circ} \mathrm{C}$ in a water tub for duration of 24 weeks. The water tub was placed in a temperature controlled environment.

\subsection{Post-ageing test methods}

Post-ageing tests were performed after each 4 weeks of immersion, both for mechanical characterization of materials and bond behavior. Changes in mechanical properties of materials were investigated by performing compressive tests on brick samples and tensile tests on epoxy resin and GFRP coupon specimens. Bond assessment tests consisted of conventional pull-off and 
pull-out tests. Five specimens were tested in each immersion period and the average results are presented next.

\subsubsection{Pull-off tests}

Pull-off tests are usually performed for evaluating the bond performance as an in-situ test method even if the results obtained represent the local adhesion strength between composite material and the substrate, and are not representative of the global bond behavior.

The pull-off tests are performed following the ASTM D4541-09 [23]. A $50 \mathrm{~mm}$ diameter partial core was drilled on the test zone with an approximate depth of $5 \mathrm{~mm}$, see Fig. 4Fig. 4 . Then, aluminum disks are glued over the GFRP surface with a high strength adhesive. The tests were carried out using a portable Proceq DYNA Z15 instrument. A constant rate of about $20 \mathrm{kPa} / \mathrm{s}$ was applied so that failure occurred within one minute. Six tests were performed for each exposure period.

\subsubsection{Single-lap shear bond test}

Single-lap shear bond tests were performed on the conditioned specimens to assess the changes in the shear bond behavior of the specimens. The tests were performed using a closed-loop servo-controlled testing machine with maximum load capacity of $50 \mathrm{kN}$. A rigid supporting steel frame was used to support the specimens appropriately and avoid misalignments in load application. The specimens were placed on the steel frame and firmly clamped to it as shown in Fig. 5 Fig. 5(a). The specimens were pulled monotonically with a speed rate of $5 \mu \mathrm{m} / \mathrm{sec}$. Tests were driven under displacement control with reference to the LVDT placed at the loaded end of the FRP composite. The resulting load was measured by means of a load cell. The relative slip between the GFRP and the masonry substrate was measured with the LVDTs glued along the 
bonded length, see Fig. 5Fig. 5(b). Two LVDTs were glued at the loaded end, two in the middle of the bonded length, and one at the free end of the FRP sheet.

\section{Results and discussion}

\subsection{Material properties}

The changes in compressive strength of brick with immersion time are shown in Fig. 6Fig. 6 . Significant reduction of compressive strength can be observed, up to $25 \%$ after 24 weeks of immersion. This reduction of the compressive strength can be due to the chemical reactions of water with brick components (dissolving effect of water) or due to insufficient firing temperature [24]. Insufficient firing temperature in clay bricks can lead to a low degree of vitrification and a high moisture expansion coefficient [25]. The degree of vitrification is defined as the amount of glass formed in the brick during firing. The water can react with the remaining clay inside the brick resulting in micro-cracking, expansion and, consequently, strength degradation.

The changes in the mechanical properties of epoxy resin, namely Young's modulus and tensile strength, are presented in Fig. 7Fig. 7. A strong reduction of mechanical properties is observed upon immersion time. The Young's modulus and tensile strength are decreased $40 \%$ and $25 \%$ after 24 weeks of immersion, corresponding to $1.2 \%$ of water absorption. It seems that the elastic modulus has reached a constant value after 12 weeks of immersion. However, the tensile strength decreases continuously during the 24 weeks of exposure. The degradation of epoxy resins due to water absorption varies with the type of epoxy resin, e.g. $[4,6,15,26]$. Tuakta and Büyüköztürk [4] reported $7 \%$ reduction of elastic modulus and $22 \%$ reduction of tensile strength in epoxy resin for $1.2 \%$ mass water absorption. In another study, Sciolti et al. [15] reported $32 \%$ reduction of tensile strength and $45 \%$ reduction of elastic modulus after 24 weeks of water immersion 
corresponding to $4 \%$ mass water absoprtion. The observed reduction in mechanical properties is due to the plasticization and possibly hydrolysis of epoxy resin [10, 27-28]. The absorbed water acts as a plasticizer, which usually reduces the $T_{\mathrm{g}}$ and mechanical properties. The decrease in the $T_{\mathrm{g}}$ reflects the degree of plasticization and occurrence of the water/resin interactions [16], but this reduction can be partially regained upon drying [29]. For long exposure periods, the possibility of resin degradation increases because hydrolytic reactions or chain scission may also occur, and these can strongly affect the resin performance [16].

The changes in mechanical properties of GFRP coupons due to water immersion are presented in Fig. 8Fig. 8. Again, Young's modulus and tensile strength decrease significantly. The Young's modulus reached a plateau after 16 weeks of immersion with $38 \%$ degradation. The tensile strength decreased about $40 \%$ after 24 weeks of exposure corresponding to $3.67 \%$ water uptake. Severe deterioration of mechanical properties of GFRP (50\% reduction in tensile strength) has also been reported in [30]. Mechanical properties of fiber reinforced polymers are usually fiber dominated. Since fibers are protected with epoxy resin no degradation is expected due to water attack. However, the formation of voids and non-uniformities in the specimens prepared following the wet lay-up procedure increases the vulnerability of the composite material to moisture uptake [26]. The observed degradation in mechanical properties of GFRP coupons can be attributed to the degrading effect of water in the epoxy and epoxy/fiber interface. Moreover, the water can attack fibers which results in corrosion and fiber/epoxy bond degradation [28].

On the other hand, the resin layers between fibers may not be polymerized completely at earlier times. Polymerization and curing take place over an extended period of time and can be accelerated due to moisture uptake resulting in an initial increase of the composite material's performance [15]. This post-curing behavior can offset the degrading effect of water attack. 
Since the specimens were cured for two months before moisture conditioning, it is assumed that the polymerization has fully occurred. Therefore, the results present solely the strength degradation due to water immersion.

\subsection{Pull-off tests}

The changes in the pull-off bond strength of the specimens are presented in Fig. 9Fig. 9 for all the tested specimens. The mean values are obtained from the average of six tests. Some scatter is found which is common in this type of test [31]. A large and progressive reduction of bond strength can be observed in the specimens with time. The pull-off bond strength has been reduced 56\% after 24 weeks of immersion. However, the failure mode remained cohesive in all immersion periods with reduction of the thickness of the detached brick layer. The thickness of the detached brick layer, being thick initially, was reduced until reaching the primer impregnated layer of the brick. The results obtained from the pull-off tests depend on the local condition of the specimens and they cannot be directly used to evaluate the bond behavior, as stated before.

\subsection{Single-lap shear bond test}

Typical load-slip curves obtained from experimental tests for different immersion times are shown in Fig. 10Fig. 10. It can be observed that the bond stiffness and strength are degraded in time. Moreover, a relatively less brittle behavior can be observed in the immersed specimens, when compared to the reference specimens. The load-slip behavior of the specimens after 24 weeks of immersion exhibits $1.4 \mathrm{~mm}$ of slip at the deboning point. In comparison, debonding has been occurred at $0.6 \mathrm{~mm}$ slip in the reference specimens. An opposite behavior was reported in [7] for CFRP-strengthened calcernite stones, where a more brittle bond behavior was found in the specimens after water immersion. 
A comparison between the slip values captured by the LVDTs at the loaded end and middle of the bonded length, see Fig. 11Fig. 11, shows that the slip at the middle is negligible in the reference specimens. In the specimens with 12 weeks of immersion, the slip at the middle is negligible initially, but it increases after the peak bond strength until complete debonding. In the specimens with 24 weeks of immersion, the slip at the middle starts to increase before reaching the maximum bond strength and it continues until complete debonding. This phenomenon, being the result of stiffness reduction in the GFRP and epoxy resin, shows an increase of effective bond length in the specimens with time due to water immersion. The effective bond length is defined as the minimum length along which the strains and stresses are transferred to the substrate. Changes in effective bond length due to water immersion, even if not considered in current design procedures, can cause unsatisfactory failure modes in the strengthened specimens. However, more precise instrumentation such as strain gauges or digital image correlation techniques is necessary for accurate measurement of the changes in the effective bond length. The changes in the debonding strength and stiffness with immersion time are presented in Fig. 12Fig. 12. The results shown provide the average of five tested specimens for each immersion period. The bond strength decreased slightly in the first 12 weeks of immersion (about 16\%). The rate of degradation increased after 12 weeks of immersion reaching $35 \%$ reduction of bond strength after 20 weeks of exposure. It seems that the degradation is less dependent on time after 20 weeks of immersion and the bond strength might have reached a residual value. However, performing water immersion tests for longer periods is necessary for obtaining a clear insight into the degradation pattern upon time. A similar residual bond strength has been reported in [7] after 8 weeks of immersion for CFRP strengthened calcernite stones. Water at the bond interface replaces the brick/adhesive contact resulting in a weaker boundary layer. The free surface energy 
at the water/epoxy interface and brick/epoxy interface is much lower than the one at the brick/epoxy. Therefore, the presence of water at the interface decreases the free surface energy of adhesion, resulting in lower fracture energy and bond strength degradation values [6].

The bond stiffness, defined as the initial slope of the bond-slip curves, has decreased significantly in the first eight weeks (about 60\%). Afterwards, the rate of degradation in the bond stiffness decreased, reaching $80 \%$ reduction in the end of the experimental program. This reduction in the bond stiffness can be attributed to the stiffness reduction in epoxy resin, GFRP, and FRP/brick bond. In comparison, the stiffness was observed to remain unaffected with immersion time in CFRP-strengthened calcernite stones [7] and concrete specimens strengthened with CFRP laminates $[3,11]$. In general, it seems that degradation diminishes with time for bond strength and stiffness. This phenomenon, being an evidence of reaching a thermodynamic balance, has also been reported in [3-4].

A drawback of strength-based approaches in investigating the environmental effects on the bond behavior, as done in Fig. 12Fig. 12, is that the results depend on the geometrical characteristics of the specimens. Considering the debonding as a local failure that involves crack propagation, fracture mechanics approaches seem to be more appropriate [4]. In fracture-based approaches, the degradation parameter is usually the fracture energy or the critical energy release rate. For generalizing the results presented, the changes in the fracture energy due to moisture ingress is determined according to CNR DT200 [32], as:

$$
G_{f}=\frac{P_{\max }^{2}}{b_{f}^{2}\left(2 E_{f} t_{f}\right)}
$$

where, $P_{\max }$ is the debonding strength, $b_{f}$ is the FRP width, $E_{f}$ is the FRP elastic modulus, and $t_{f}$ is the FRP thickness. 
Fig. 13Fig. 13 shows that in the first 12 weeks of exposure there is no degradation in the interfacial fracture energy. However, significant degradation occurs after 12 weeks and it continues until 20 weeks of immersion. It seems that after 20 weeks of immersion, the fracture energy has reached a residual value with $40 \%$ degradation comparing to its initial value. A similar degradation trend has also been reported in [3]. A cohesive failure mode was mainly observed in the specimens, see Fig. 14Fig. 14. However, the thickness of the detached layer decreased with time up to a thin layer equal to the primer impregnated thickness. Generally, the fracture surface has moved to the brick/primer interface with the increase of water content at the interface, as reported in [3, 6-7].

\section{Conclusions}

The results of an experimental investigation about the effect of water on the bond behavior in FRP-strengthened masonry bricks was presented, demonstrating that:

- water immersion resulted in a reduction of mechanical properties of material constituents. Compressive strength of masonry bricks reduced $25 \%$ after 24 weeks of water immersion. A similar reduction was also observed in the tensile strength of epoxy resin. However, the reduction in tensile strength of GFRP fabric was $40 \%$ at the end of ageing, which is due to the reduction in the mechanical properties of epoxy resin and the degradation of epoxy/fiber interfaces. The elastic modulus of epoxy resin and GFRP fabric also decreased with time, about $40 \%$ after 24 weeks of water immersion.

- the pull-off strength of the specimens decreased significantly (about 60\%) after 24 weeks of immersion. However, the results obtained from pull-off tests are not completely consistent with the shear bond tests, where a different degradation trend was observed. The failure mode 
remained cohesive in time but the fracture surface moved from the brick to the brick/primer interface.

- shear bond tests showed that the ductility of the bond behavior increases with immersion time in the tested specimens. The bond strength and stiffness were observed to decrease, while the debonding slip increased. It was observed that the degradation in the bond strength and stiffness diminishes with time and possibly a residual value is obtained after a certain immersion time. However, performing water immersion tests for longer periods is necessary for having a clear idea about the degradation trend upon time. A similar degradation was also observed in interfacial fracture energy. The failure mode was cohesive in all the specimens, with the fracture surface inside the brick. However, the fracture surface moved towards the brick/primer interface with time.

\section{Acknowledgements}

This work was partly funded by project FP7-ENV-2009-1-244123-NIKER of the 7th Framework Program of the European Commission, which is gratefully acknowledged. The first author also acknowledges the financial support of the Portuguese Science Foundation (Fundação de Ciência e Tecnologia, FCT), through grant SFRH/BD/80697/2011.

\section{References}

[1] Hollaway LC. A review of the present and future utilisation of FRP composites in the civil infrastructure with reference to their important in-service properties. Constr Build Mater. 2010;24(12):2419-45. 
[2] Karbhari VM, Chin W, Hunston D, Benmokrane B, Juska T, Morgan R, et al. Durability gap analysis for fiber-reinforced polymer composites in civil infrastructures. J Compos Constr. $2003 ; 7(3): 238-47$.

[3] Ouyang Z, Wan B. Experimental and numerical study of water effect on bond fracture energy between FRP and concrete in moist environments. J Reinf Plast Compos. 2008;27(2):205-23.

[4] Tuakta C, Büyüköztürk O. Deterioration of FRP/concrete bond system under variable moisture conditions quantified by fracture mechanics. Compos Part B. 2011;42(2):145-54.

[5] Frigione M, Aiello MA, Naddeo C. Water effects on the bond strength of concrete/concrete adhesive joints. Constr Build Mater. 2006;20(10):957-70.

[6] Wan B, Petrou MF, Harries KA. Effect of the presence of water on the durability of bond between CFRP and concrete. J Reinf Plast Compos. 2006;25(8):875-90.

[7] Sciolti MS, Aiello MA, Frigione M. Influence of water on bond behavior between CFRP sheet and natural calcareous stones. Compos Part B. 2012;43(8):3239-50.

[8] Khoshbakht M, Lin MW, Berman JB. Analysis of moisture-induced stresses in an FRP composites reinforced masonry structure. Finite Elem Anal Des. 2006;42(5):414-29.

[9] Khoshbakht M, Lin MW. A finite element model for hygro-thermo-mechanical analysis of masonry walls with FRP reinforcement. Finite Elem Anal Des. 2010;46(10):783-91.

[10] Lau D, Büyüköztürk O. Fracture characterization of concrete/epoxy interface affected by moisture. Mech Mater. 2010;42(12):1031-42.

[11] Ouyang Z, Wan B. Nonlinear deterioration model for bond interfacial fracture energy of FRP-concrete joints in moist environments. J Compos Constr. 2009;13(1):53-63. 
[12] Lourenço PB, Fernandes F, Castro F. Handmade clay bricks: Chemical, physical and mechanical properties. Int J Archit Herit. 2010;41(1):38-58.

[13] EN 772-1. Methods of test for masonry units -Part 1: Determination of compressive strength; 2002.

[14] UNI EN 8942-3. Clay bricks and blocks. Test methods; 1986.

[15] Sciolti MS, Frigione M, Aiello MA. Wet lay-up manufactured FRPs for concrete and masonry repair: influence of water on the properties of composites and on their epoxy components. J Compos Constr. 2010;14(6):823-33.

[16] Frigione M, Lettieri M, Mecchi AM. Environmental effects on epoxy adhesives employed for restoration of historical buildings. J Mater Civil Eng. 2006;18(5):715-22.

[17] EN ISO 527-1. Plastics-determination of tensile properties- Part 1: general principles; 1993.

[18] ASTM D7565-10. Standard test method for determining tensile properties of fiber reinforced polymer matrix composites used for strengthening of civil structures; 2010.

[19] Cromwell JR, Harries KA, Shahrooz BM. Environmental durability of externally bonded FRP materials intended for repair of concrete structures. Constr Build Mater. 2011;25(5):252539.

[20] ASTM D570-98(2010)e1. Standard test method for water absorption of plastics; 2010.

[21] Karbhari VM, Ghosh K. Comparative durability evaluation of ambient temperature cured externally bonded CFRP and GFRP composite system for repair of bridges. Compos Part A. 2009;40(9):1353-63.

[22] Frigione M, Lettieri M. Procedures conditioning the absorption/desorption behavior of coldcured epoxy resins. J Polym Sci B Polym Phys. 2008;46(3):1320-36. 
[23] ASTM D4541. Standard test method for pull-off adhesion strength of coatings using portable adhesion testers; 2009.

[24] Abanilla M, Li Y, Karbhari VM. Durability characterization of wet layup graphite/epoxy composites used in external strengthening. Compos Part B. 2006;37(2/3):200-12.

[25] Moy P, Karasz F. Epoxy-water interactions. Polym Eng Sci. 1980;20(4):315-9.

[26] Carol L, Schutte CL. Environmental durability of glass-fiber composites. Mater Sci Eng A. 1994;13(7):265-323

[27] Zhou J, Lucas J. Hygrothermal effects of epoxy resins. Part II: variations of glass transition temperature. Polym. 1999;40(20):5513-22.

[28] Tavakkolizadeh M, Saadatmanesh H. Environmental effects on tensile properties of FRP laminates made using wet-lay-up method. . In: L. C. Hollaway MKCaSSJM, editor. Proceedings of the Second Inter Conf Adv Polym Compos Struct Applic Constr. University of Surrey, Guilford, U.K.2004. p. 619-32.

[29] Bonaldo E, Barros JO, Lourenço P. Bond characterization between concrete substrate and repairing SFRC using pull-off testing. Int J Adhes Adhes. 2005;25(6):463-74.

[30] CNR-DT 200. Guide for the design and construction of externally bonded FRP systems for strengthening existing structures. CNR-DT 200/2004. Rome: National Research Council; 2004. 


\section{List of Tables}

Table 1. Material properties.

\section{List of Figures}

Fig. 1. Specimens used for material testing (dimensions in $\mathrm{mm}$ ): (a) Brick under compression; (b) brick under flexural tension; (c) epoxy resin and primer under tension; (d) GFRP coupon under tension.

Fig. 2. Moisture uptake behavior of materials constituents: (a) brick; (b) epoxy resin and GFRP coupon.

Fig. 3. Specimens' details (dimensions in mm): (a) pull-off test specimen; (b) single-lap shear bond test specimen.

Fig. 4. Pull-off tests setup.

Fig. 5. Single-lap shear bond tests: (a) test setup (perspective); (b) specimen instrumentation (front view).

Fig. 6. Compressive strength of brick under continuous water immersion.

Fig. 7. Mechanical properties of epoxy under continuous water immersion: (a) modulus of elasticity; (b) tensile strength.

Fig. 8. Mechanical properties of GFRP coupons under continuous water immersion: (a) elastic modulus; (b) tensile strength.

Fig. 9. Pull-off bond strength under continuous water immersion.

Fig. 10. Envelope and average load-slip curves obtained from single-lap shear bond tests.

Fig. 11. Typical load-slip behavior at the loaded end and middle bonded length, through time.

Fig. 12. Debonding behavior under continuous water immersion: (a) debonding force; (b) bond stiffness.

Fig. 13. Changes in the average interfacial fracture energy due to water immersion.

Fig. 14. Failure mode of the specimens in shear bond tests. 
Table 1. Material properties (five specimens).

\begin{tabular}{lcrr}
\hline Masonry brick & & Average & CoV $(\%)$ \\
\hline Compressive strength & $f_{\mathrm{cb}}(\mathrm{MPa})$ & 9.10 & 15.7 \\
Flexural tensile strength & $f_{\mathrm{tb}}(\mathrm{MPa})$ & 1.54 & 24.6 \\
Elastic modulus & $E_{\mathrm{b}}(\mathrm{GPa})$ & 1.73 & 21.4 \\
\hline GFRP coupons & & & \\
\hline Tensile strength & $f_{\mathrm{tf}}(\mathrm{MPa})$ & 1250 & 15.0 \\
Elastic modulus & $E_{\mathrm{f}}(\mathrm{GPa})$ & 79.2 & 6.8 \\
Ultimate deformation & $\varepsilon(\%)$ & 1.86 & 20.2 \\
\hline Epoxy resin & & & \\
\hline Tensile strength & $f_{\mathrm{tm}}(\mathrm{MPa})$ & 53.8 & 9.7 \\
Elastic modulus & $E_{\mathrm{m}}(\mathrm{GPa})$ & 2.52 & 9.5 \\
Glass transition temperature & $T_{\mathrm{g}}(\mathrm{C})$ & 70.0 & 3.2 \\
(four specimens only) & & & \\
Primer & $f_{\mathrm{tm}}(\mathrm{MPa})$ & 34.4 & 11.1 \\
\hline Tensile strength & $E_{\mathrm{m}}(\mathrm{GPa})$ & 2.36 & 6.1 \\
Elastic modulus & &
\end{tabular}




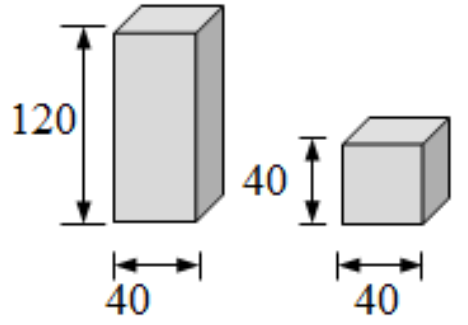

(a)

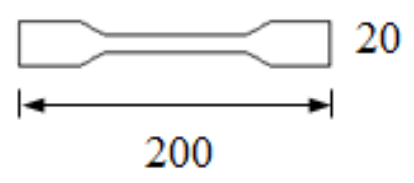

(c)

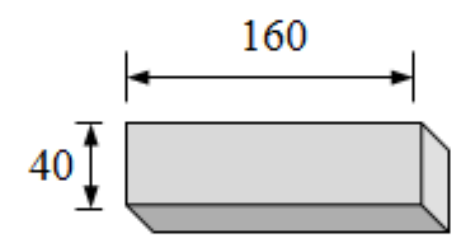

(b)

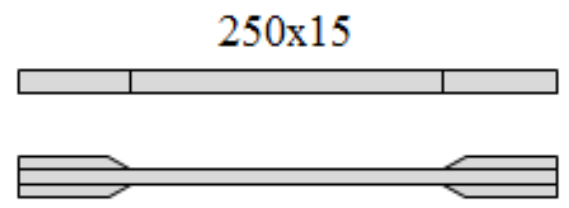

(d)

Fig. 1. Specimens used for material testing (dimensions in mm): (a) Brick under compression; (b) brick under flexural tension; (c) epoxy resin and primer under tension; (d) GFRP coupon under tension. 


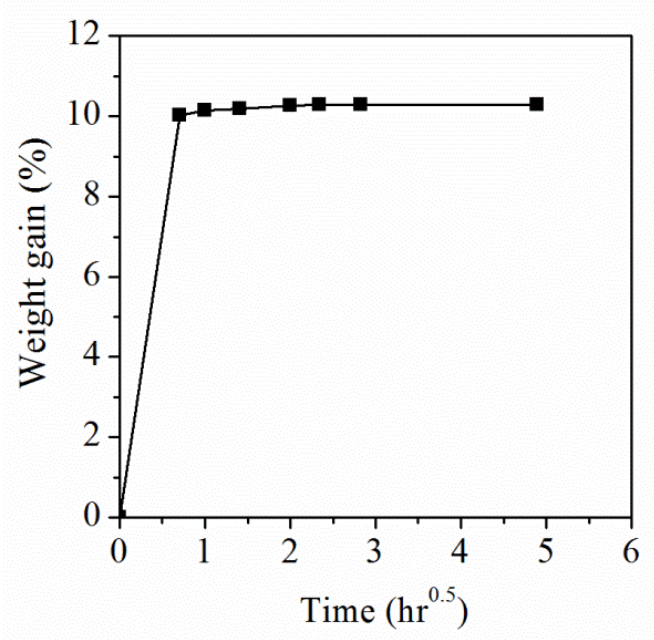

(a)

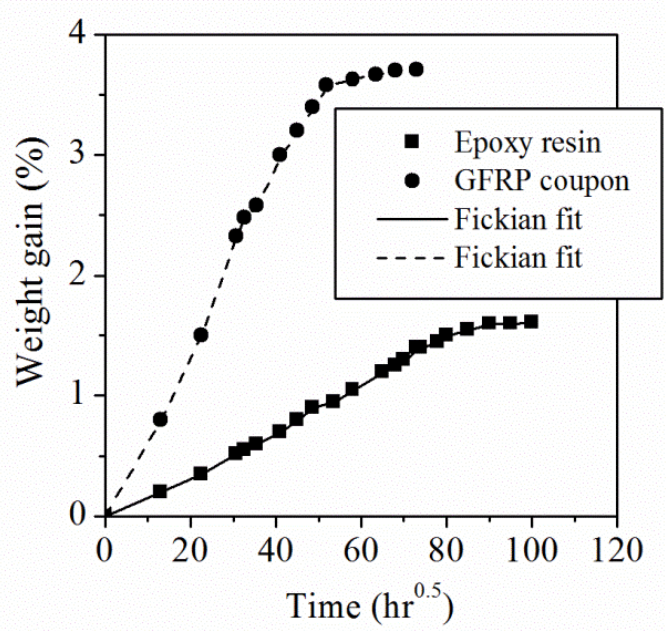

(b)

Fig. 2. Moisture uptake behavior of materials constituents: (a) brick; (b) epoxy resin and GFRP coupon. 

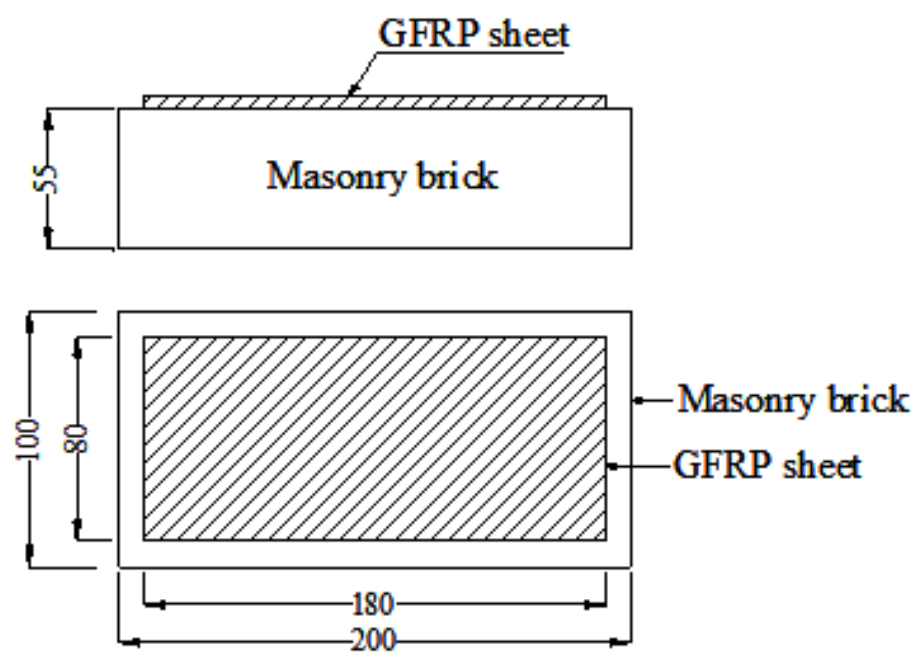

(a)

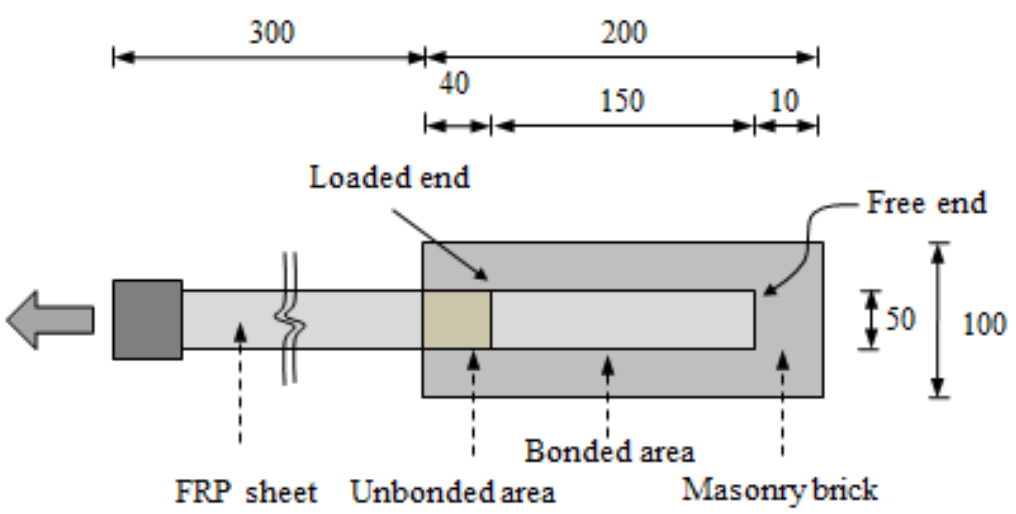

(b)

Fig. 3. Specimens' details (dimensions in mm): (a) pull-off test specimen; (b) single-lap shear bond test specimen. 

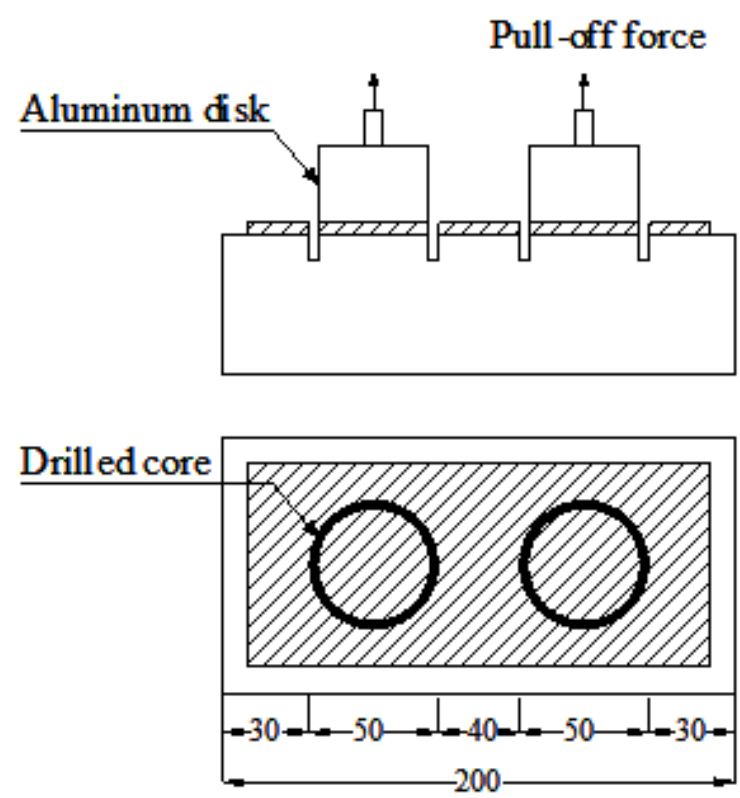

Fig. 4. Pull-off tests setup. 


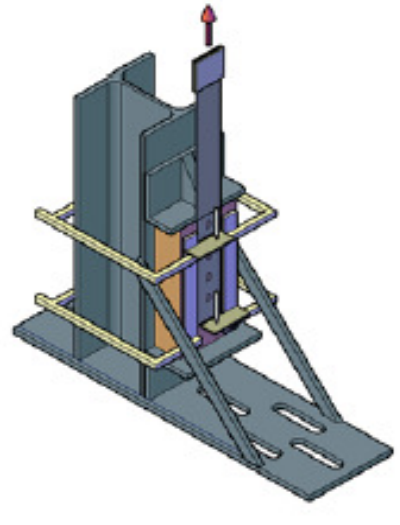

(a)

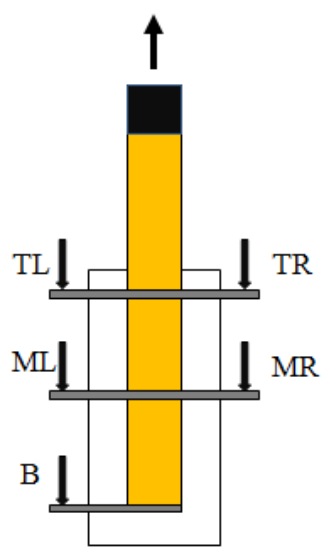

(b)

Fig. 5. Single-lap shear bond tests: (a) test setup (perspective); (b) specimen instrumentation (front view). 


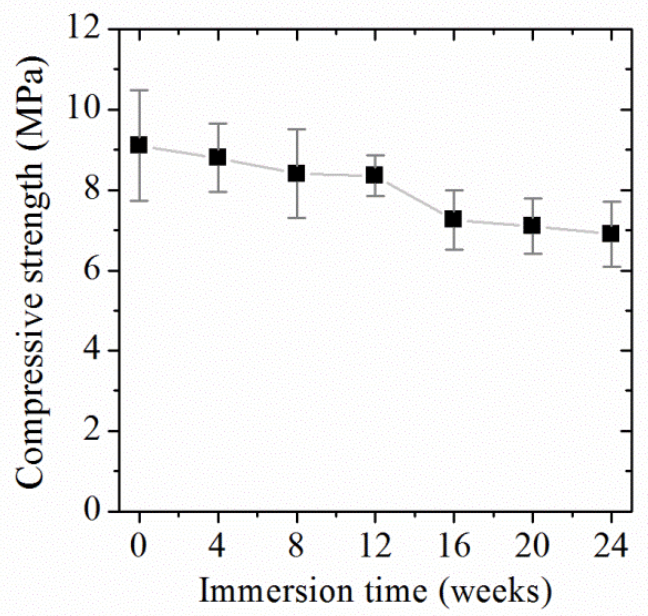

Fig. 6. Compressive strength of brick under continuous water immersion. 


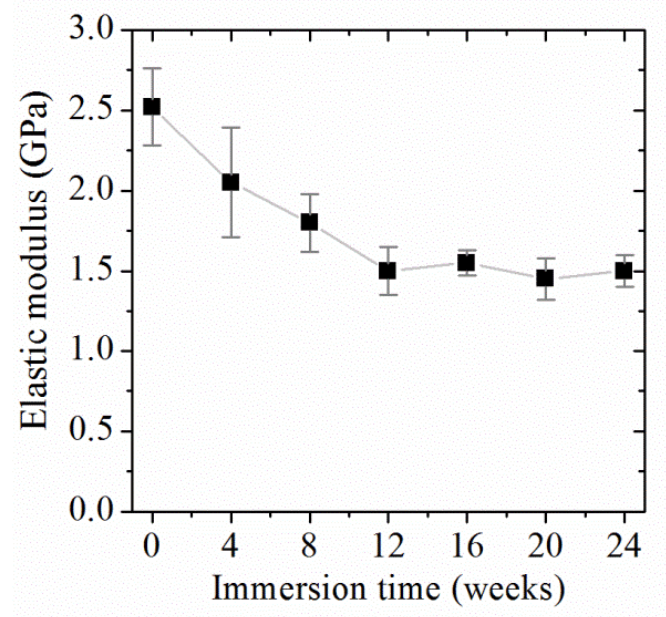

(a)

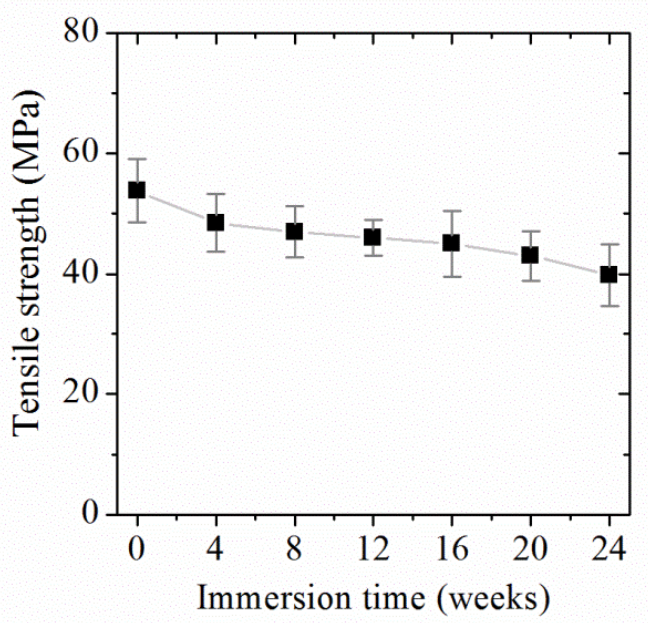

(b)

Fig. 7. Mechanical properties of epoxy under continuous water immersion: (a) modulus of elasticity; (b) tensile strength. 


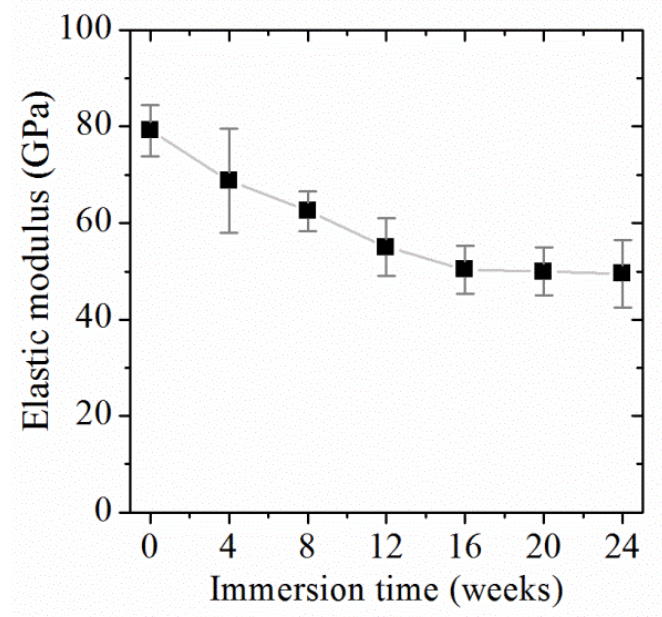

(a)

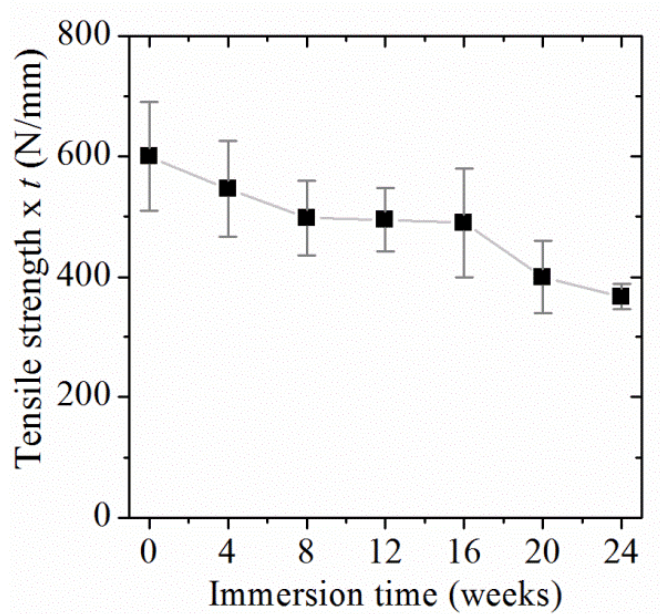

(b)

Fig. 8. Mechanical properties of GFRP coupons under continuous water immersion: (a) elastic modulus; (b) tensile strength. 


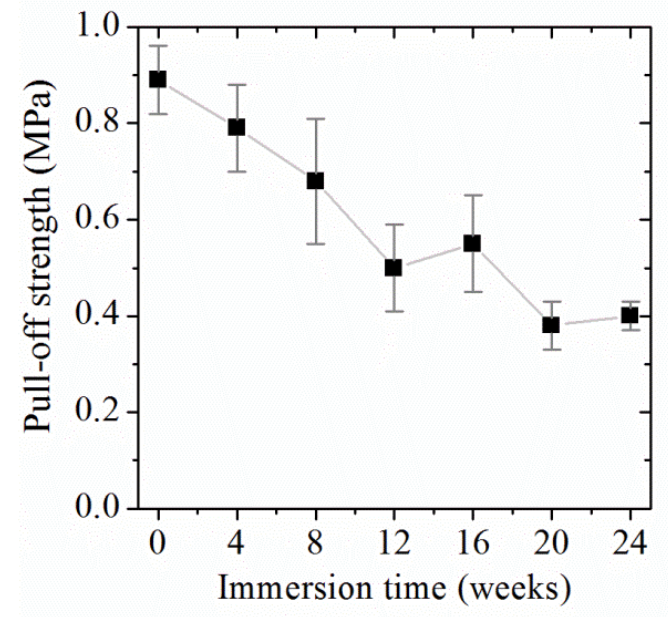

Fig. 9. Pull-off bond strength under continuous water immersion. 

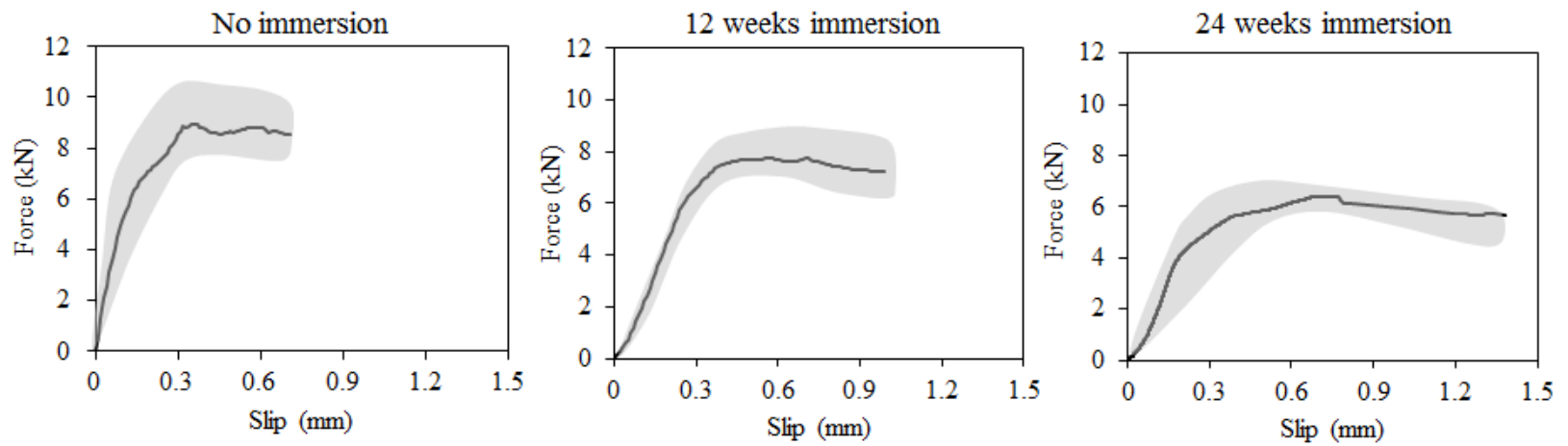

Fig. 10. Envelope and average load-slip curves obtained from single-lap shear bond tests. 

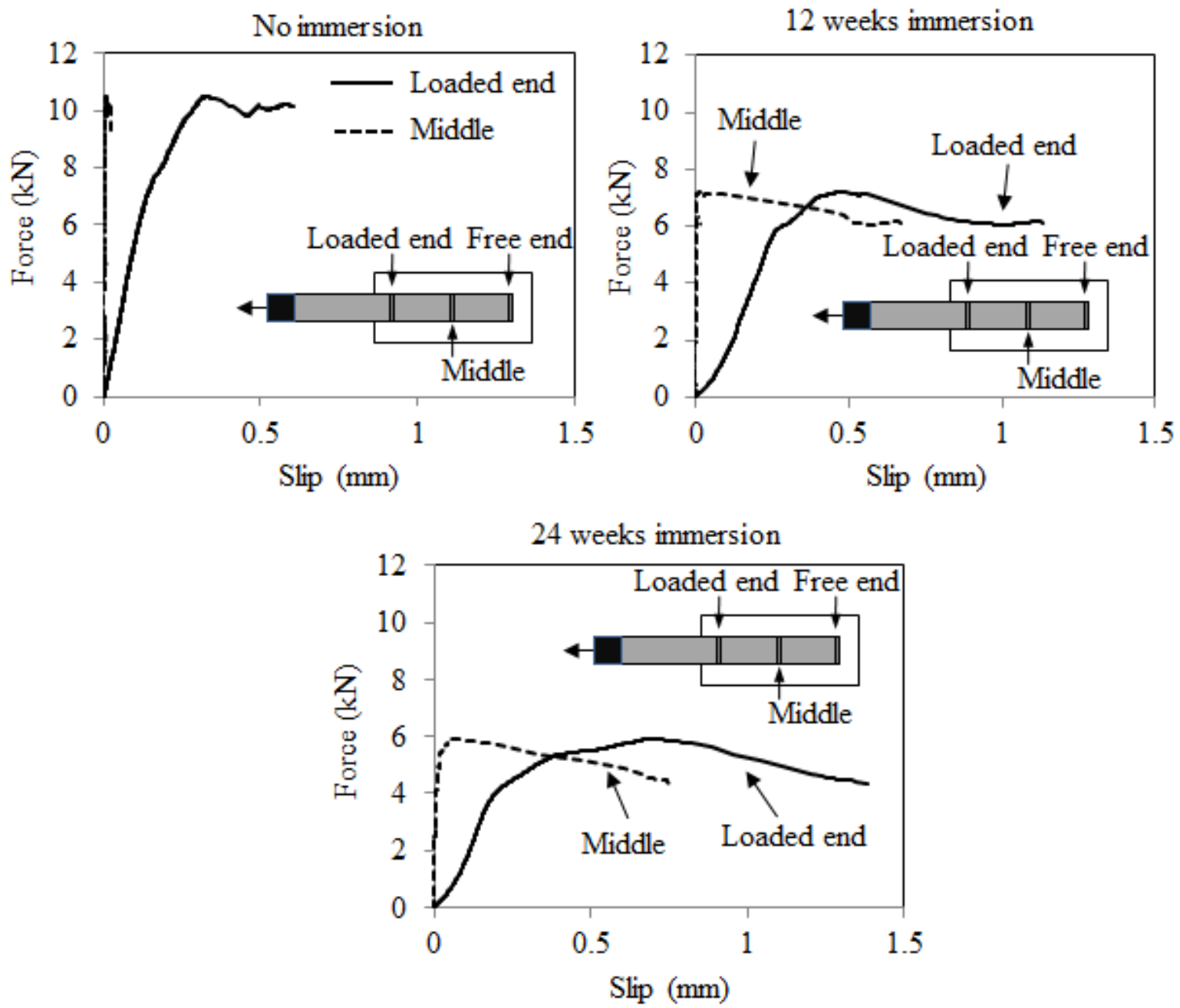

Fig. 11. Typical load-slip behavior at the loaded end and middle bonded length, through time. 


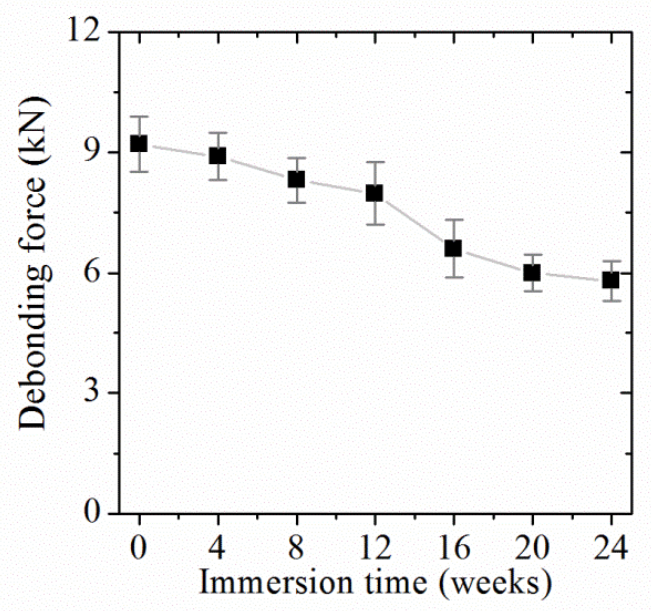

(a)

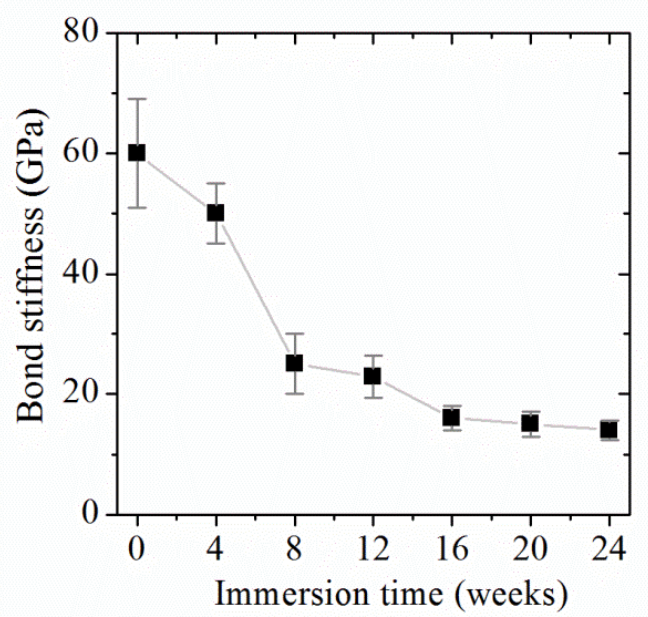

(b)

Fig. 12. Debonding behavior under continuous water immersion: (a) debonding force; (b) bond stiffness. 


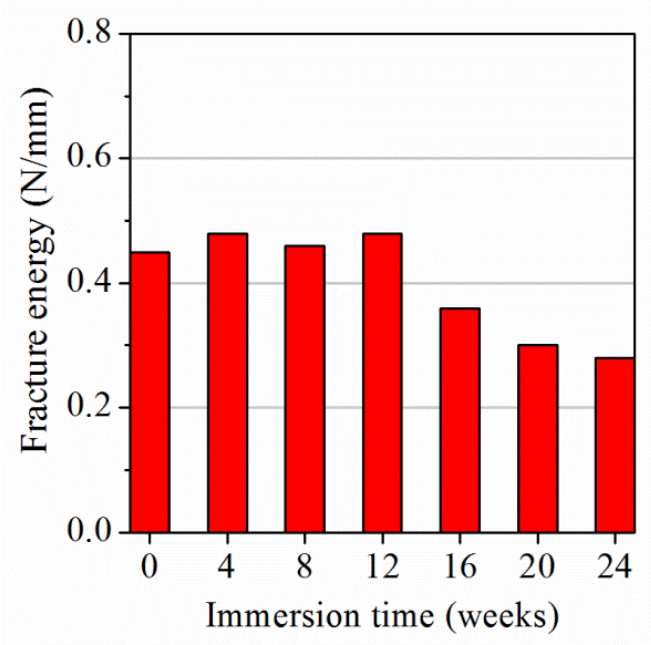

Fig. 13. Changes in the average interfacial fracture energy due to water immersion. 
No immersion

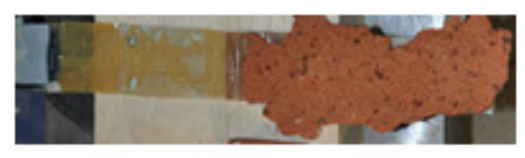

12 weeks immersion

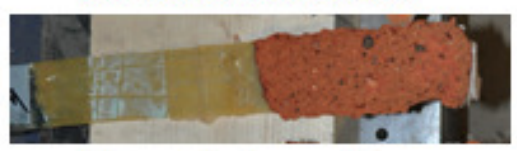

24 weeks immersion

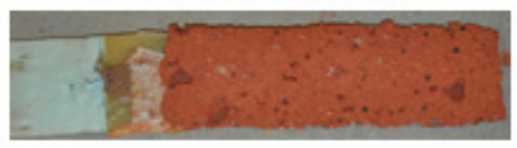

Fig. 14. Failure mode of the specimens in shear bond tests.

[1] Hollaway LC. A review of the present and future utilisation of FRP composites in the civil infrastructure with reference to their important in-service properties. Constr Build Mater. 2010;24(12):2419-45.

[2] Karbhari VM, Chin W, Hunston D, Benmokrane B, Juska T, Morgan R, et al. Durability gap analysis for fiber-reinforced polymer composites in civil infrastructures. J Compos Constr. 2003;7(3):238-47.

[3] Ouyang Z, Wan B. Experimental and numerical study of water effect on bond fracture energy between FRP and concrete in moist environments. J Reinf Plast Compos. 2008;27(2):205-23.

[4] Tuakta C, Buyukozturk O. Deterioration of FRP/concrete bond system under variable moisture conditions quantified by fracture mechanics. Composites: Part B. 2011;42(2):145-54.

[5] Frigione M, Aiello MA, Naddeo C. Water effects on the bond strength of concrete/concrete adhesive joints. Construction and Building Materials. 2006;20(10):957-70.

[6] Wan B, Petrou MF, Harries KA. Effect of the presence of water on the durability of bond between CFRP and concrete. J Reinf Plast Compos. 2006;25(8):875-90.

[7] Sciolti MS, Aiello MA, Frigione M. Influence of water on bond behavior between CFRP sheet and natural calcareous stones. Compos Part B. 2012;43(8):3239-50.

[8] Khoshbakht M, Lin MW, Berman JB. Analysis of moisture-induced stresses in an FRP composites reinforced masonry structure. Finite Elements in Analysis and Design.

2006;42(5):414-29.

[9] Khoshbakht M, Lin MW. A finite element model for hygro-thermo-mechanical analysis of masonry walls with FRP reinforcement. Finite Elem Anal Des. 2010;46(10):783-91.

[10] Lau D, Buyukozturk O. Fracture characterization of concrete/epoxy interface affected by moisture. Mech Mater. 2010;42(12):1031-42.

[11] Ouyang Z, Wan B. Nonlinear deterioration model for bond interfacial fracture energy of FRP-concrete joints in moist environments. Journal of Composites for Construction.

2009;13(1):53-63. 
[12] Lourenço PB, Fernandes F, Castro F. Handmade clay bricks: Chemical, physical and mechanical properties. Int J Archit Herit. 2010;41(1):38-58.

[13] EN 772-1. Methods of test for masonry units -Part 1: Determination of compressive strength. 2002.

[14] UNI EN 8942-3. Clay bricks and blocks. Test methods. 1986.

[15] Sciolti MS, Frigione M, Aiello MA. Wet lay-up manufactured FRPs for concrete and masonry repair: influence of water on the properties of composites and on their epoxy components. Journal of Composites for Construction. 2010;14(6):823-33.

[16] Frigione M, Lettieri M, Mecchi AM. Environmental effects on epoxy adhesives employed for restoration of historical buildings. J Mater Civil Eng. 2006;18(5):715-22.

[17] EN ISO 527-1. Plastics-determination of tensile properties- Part 1: general principles. 1993.

[18] ASTM D7565-10. Standard test method for determining tensile properties of fiber reinforced polymer matrix composites used for strengthening of civil structures. 2010.

[19] Cromwell JR, Harries KA, Shahrooz BM. Environmental durability of externally bonded FRP materials intended for repair of concrete structures. Constr Build Mater. 2011;25(5):252539.

[20] ASTM D570 - 98(2010)e1 Standard Test Method for Water Absorption of Plastics. ASTM; 2010.

[21] Karbhari VM, Ghosh K. Comparative durability evaluation of ambient temperature cured externally bonded CFRP and GFRP composite system for repair of bridges. Composites: Part A. 2009;40(9):1353-63.

[22] Frigione M, Lettieri M. Procedures conditioning the absorption/desorption behavior of coldcured epoxy resins. J Polym Sci B Polym Phys. 2008;46(3):1320-36.

[23] ASTM D4541. Standard tests method for pull-off adhesion strength of coatings using portable adhesion testers. 2009.

[24] Cultrone G, De la Torre MJ, Sebastian EM, Cazalla O, Rodriguez-Navarro C. Behavior of Brick Samples in Aggressive Environments. Water, Air, Soil Pollut. 2000;119(1-4):191-207.

[25] Elert K, Cultrone G, Navarro CR, Pardo ES. Durability of bricks used in the conservation of historic buildings- influence of composition and microstructure. Journal of Cultural Heritage. 2003;4(2):91-9.

[26] Abanilla M, Li Y, Karbhari VM. Durability characterization of wet layup graphite/epoxy composites used in external strengthening. Composites B. 2006;37(2/3):200-12.

[27] Moy P, Karasz F. Epoxy-water interactions. Polym Eng Sci. 1980;20(4):315-9.

[28] Carol L, Schutte CL. Environmental durability of glass-fiber composites. Mater Sci Eng A. 1994;13(7):265-323.

[29] Zhou J, Lucas J. Hygrothermal effects of epoxy resins. Part II: variations of glass transition temperature. Polymer. 1999;40(20):5513-22.

[30] Tavakkolizadeh M, Saadatmanesh H. Environmental effects on tensile properties of FRP laminates made using wet-lay-up method. . In: L. C. Hollaway MKCaSSJM, editor. Proceedings of the Second International Conference on Advanced Polymer Composites for Structural Applications in Construction. University of Surrey, Guilford, U.K.2004. p. 619-32. 
[31] Bonaldo E, Barros JO, Lourenco P. Bond characterization between concrete substrate and repairing SFRC using pull-off testing. Int J Adhes Adhes. 2005;25(6):463-74.

[32] CNR-DT 200. Guide for the design and construction of externally bonded FRP systems for strengthening existing structures. CNR-DT 200/2004. Rome: National Research Council; 2004. 